\title{
Foreword
}

\section{Moving Beyond the Ordinary: \\ Transcending Traditions in \\ Pediatric Anesthesia}

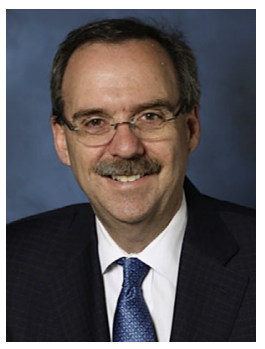

Lee $A$. Fleisher, MD

Consulting Editor

Surgical and procedural interventions in complex pediatric patients have evolved greatly over the years. Many of these patients undergo surgery at children's hospitals, but they could present in any hospital setting, and therefore, knowledge of their care is important for a large number of anesthesiologists. In addition, patients with congenital heart disease can survive into adulthood and require further interventions, and therefore, all anesthesiologists need to understand the care of the adult patient with congenital heart disease. In this issue of Anesthesiology Clinics, the editors have brought together a series of articles that cover a broad array of pediatric anesthesia topics that would be of interest to a wide variety of anesthesiologists.

This issue was edited by rising pediatric leaders from two of the preeminent pediatric hospitals in the United States. Vanessa A. Olbrecht, MD, MBA is an Associate Professor of Anesthesiology at Cincinnati Children's Hospital Medical Center and an outcomes researcher. She is also Director of Quality Improvement and Accreditation for Procedural Sedation. Alison R. Perate, MD is Assistant Professor of Anesthesiology at the University of Pennsylvania and The Children's Hospital of Philadelphia. She is also 
Associate Director of the Trauma Center. Together they have assembled an amazing group of leaders in pediatric anesthesia to describe unique practices.

Lee A. Fleisher, MD Perelman School of Medicine University of Pennsylvania 3400 Spruce Street, Dulles 680

Philadelphia, PA 19104, USA

E-mail address:

Lee.Fleisher@uphs.upenn.edu 